\title{
Introduction to the special issue on Next Generation Network Architectures, Protocols, Theory, Systems and Applications
}

() Tsinghua University Press and Springer-Verlag Berlin Heidelberg 2013

This special issue of Networking Science focuses on Next Generation Network (NGN) that enables the deployment of access independent services over converged fixed and mobile networks. NGN is a packet-based network and uses the Internet protocol (IP) to transport the various types of traffic (voice, video, data and signalling). NGN facilitates easy adoption of distributed computing applications by providing high speed connectivity in a converged networked environment. It also makes end user devices and applications highly intelligent and efficient by empowering them with programmability and remote configuration options. However, there are a number of important challenges in provisioning next generation network technologies in a converged communication environment. Some preliminary challenges include those that relate to QoS, switching and routing, management and control, and security which must be addressed on an urgent or emergency basis. The consideration of architectural issues in the design and provision of secure services for NGN deserves special attention and hence is the main theme of this special issue.

Submissions to this special issue were open to original, high quality contributions that were not published or currently under review by any other archival journals or peer-reviewed conferences with formal proceedings. The authors of the papers presented at The 4th International Conference on Security of Information and Networks (SIN 2011) were especially invited to submit papers which were relevant to the main theme of the special issue. SIN 2011 was held in Sydney, Australia in November, 2011, organized jointly by Macquarie University, Süleyman Demirel University, Southern Federal University, and Technical University of Darmstadt; it provided a regional focus on aspects of information and communications security and was therefore closely aligned with the main theme of the special issue. Several SIN 2011 authors responded to the call for papers and submitted the revised and substantially extended versions of their papers; several other authors from diverse parts of the world have also responded to the call. Each submission was sent to two reviewers who were experts in different aspects of NGN. After two rounds of reviewing, four high quality papers were accepted for publication in the special issue, all in the area of security for NGN.

The carefully selected papers address the design and provision of secure services for NGN including confidentiality, identity spoofing and intrusion detection, and secure protocol design. The list of the papers included in the special issue is provided below:

(1) The paper, Generating attack scenarios for systems security validation by Anderson Morais, Iksoon Hwang, Ana Rosa Cavalli, and Eliane Martins, discusses the design and implementation of an innovative attack and script injection approach to evaluate the security of NGN based communication system. They demonstrate the viability of this approach by applying it to WAP WTLS protocol.

(2) The paper, On link encryption against wiretapping attack in network coding by Zhaohui Tang, proposes an admissible link encryption scheme for linear network coding. The author proves efficiency of the proposed scheme over traditional approach by demonstrating comparatively lower computational costs and smaller key sizes.

(3) The paper, Detection of neighbor discovery protocol based attacks in IPv6 network by Ferdous A. Barbhuiya, Gunjan Bansal, Niteesh Kumar, Santosh Biswas, and Sukumar Nandi, proposes a technique that deploys an active probing mechanism to detect all possible attacks on Network Discovery Protocol-a feature of next generation Internet protocol-IPv6. The proposed approach is detection based and has a number of advantages over prevention based schemes that are currently in use.

(4) The paper, IDS for ARP spoofing using LTL based discreet event system framework by Mahasweta Mitra, Prithu Bannerjee, Ferdous A. Barbhuiya, Santosh Biswas, and Sukumar Nandi, proposes a novel linear temporal logic (LTL) based active discrete event system to detect address resolution protocol (ARP) based spoofing attacks. The proposed scheme can also be applied to all attacks which arise because of unauthenticated messages. 
Many individuals contributed to this special issue. First and foremost, we express our gratitude to Professor David Lee, Co-Editor-in-Chief of Networking Science, for his continuing support and encouragement for the special issue. Thanks are also due to Jie Zeng, Editorial Director, for her enthusiasm and effective assistance during the review and publication stages of the special issue. We are grateful to the authors who responded to the invitation to

\section{Guest Editors}

\section{Mehmet A. Orgun}

Department of Computing

Macquarie University, Australia

\section{Josef Pieprzyk}

Department of Computing

Macquarie University, Australia

\section{Atilla Elçi}

Department of Electrical \& Electronics Engineering Aksaray University, Turkey submit their papers, and indebted to our reviewers who read the papers and provided extensive and constructive comments for the authors.

To conclude, editing this special issue has been a very rewarding experience to us. We trust that the papers published in this special issue will generate further interest in NGN, ranging from theoretical to practical issues and to their eventual deployment.

\section{Rajan Shankaran}

Department of Computing

Macquarie University, Australia

\section{Ming Xu}

Department of Networks Engineering

National University of Defense Technology, China

\section{Huaxiong Wang}

Division of Mathematical Sciences

Nanyang Technological University, Singapore 